Session 1532

\title{
Reintroducing Amateur Radio In ECE Capstone Design Projects
}

\author{
Dennis Silage \\ Electrical and Computer Engineering \\ College of Engineering, Temple University
}

\begin{abstract}
Presented here from experience are the salient steps for the reintroduction of Amateur Radio into the capstone design project in Electrical and Computer Engineering and diverse examples of such projects. These steps include the involvement of the faculty supervisor, the establishment of an Amateur Radio station as a communications laboratory sponsored by the department, a survey of the technical resources available and immediate past, current and future areas for capstone design projects utilizing Amateur Radio.
\end{abstract}

\section{It's Not Just for Morse Code Anymore}

Capstone design projects in Electrical and Computer Engineering (ECE) are an important component of the undergraduate educational process that synthesizes course material into a relevant experience. Many such projects utilize embedded software and hardware and wired, and now low power and low range wireless, data communication to produce a system. A plethora of process control robotics projects often seem to be the result.

What is not used routinely, though, is a concentration that, at one time, was quite significant in the technical development of electrical engineers in electronic communication: Amateur Radio. Significant past developments derived from amateur radio graduate student experimentation include the helix antenna by John Kraus while at Ohio University (W8PZS) and slow-scan television (SSTV) by Ralph Taggart while at the Michigan State University (W8SH).

The reasons for this void are perplexing, since the incorporation of Amateur Radio into the ECE capstone design project is without any major impediments to either the faculty supervisor, the department or the undergraduate students. The perceived impediments seem to be the requirement for an Amateur Radio license and what are the resources available and opportunities for design for the faculty supervisor and the ECE capstone design team.

Amateur Radio is a service administered by the Federal Communication Commission (FCC, $w w w . f c c . g o v)$ and as such requires a license by written examination. However, the seemingly daunting component to copy and send Morse Code has now been removed entirely for the entrylevel license required for experimentation in the frequency range for digital data and satellite communication projects. Even the maximum requirement in Morse Code proficiency for an advanced license is now only five words per minute. Although the technical and regulatory questions of the examination remain, they are quite reasonable for both the faculty supervisor 
and the undergraduate students. In fact, this exercise provides a ECE capstone design experience in regulatory issues and licensing.

Amateur Radio provides unique and interesting opportunities for the ECE capstone design course. Low earth orbiting Amateur Radio satellites have been available since 1961 and provide significant design experience in polarized antennas, automated tracking, RF modem design, and data analysis. Various modulation and demodulation methods for digital data communication in fading channels are implemented on architectures as diverse as a digital signal processing (DSP) microcomputer or a PC with a sound card peripheral. Low bit rate Amateur Radio digital voice standards are being developed to replace traditional analog modulation methods.

Unlike in the commercial sector, successful capstone design projects in Amateur Radio for ECE undergraduates can even have the unique opportunity to add significantly to the base of knowledge and be presented in a refereed technical forum, such as the yearly Digital Communications Conference (www.tapr.org or www.arrl.org), or in an Amateur Radio publication such as $Q E X^{1}$, which is a forum for communications experimenters.

\section{Amateur Radio: All Resources}

The American Radio Relay League (ARRL, www.arrl.org), established in 1914, is the national association for Amateur Radio. The ARRL publishes study guides for the theory and regulatory questions encountered in the entry-level through advanced license examinations. The Amateur Radio entry-level license has even been attained by adolescences after appropriate study.

Another Amateur Radio resource is the Tucson Amateur Packet Radio organization (TAPR, $w w w$.tapr.org). The original technical contribution of TAPR was the development of the $A X .25$ packet radio data protocol over two decades ago, which was adapted from the $X .25$ standard for Amateur Radio use. Amateur Radio operators thus had developed complex wireless data communication systems with store and forward and self-organization more than a decade before the IEEE 802.11 standard. TAPR provides reference materials, technical forums and supervisory organization for a variety of Amateur Radio technical projects.

Some of these projects include the integration of the $A X .25$ packet radio data protocol and the Global Positioning System (GPS) for radiolocation, a decade before the inclusion of this technology in fifth-generation cellular telephony, and the software-defined radio. Amateur Radio projects are, by definition, non-commercial and open-source and are thus eligible for an innovative contribution even by undergraduate students in an ECE capstone design course.

Amateur Radio technical resources for the capstone design project include reference texts, websites, and conferences. The ARRL and TAPR also publish a wide variety of technical publications, which are valuable resources for an ECE capstone design project, and only a few of which can be described here.

The ARRL Handbook ${ }^{2}$, published yearly, is a compendium of fundamental electrical and electronic theory from DC to DSP and practical designs and projects in analog and digital communications. The ARRL Spread Spectrum Sourcebook ${ }^{3}$ chronicles the over two decades of 
Amateur Radio experimentation in frequency hopping and direct sequence spread spectrum (FHSS and DSSS).

The Radio Amateur's Satellite Handbook ${ }^{4}$ describes satellite communication that can be an interesting and multifaceted component of an ECE capstone design project. The ARRL text Digital Signal Processing Technology ${ }^{5}$ describes communication signal processing and the emerging Amateur Radio experience with the software-defined radio concept.

The TAPR text Wireless Digital Communications: Design and Theory ${ }^{6}$ presents a concise overview of topical materials in digital data communications quite suitable for ECE undergraduate students. Finally, the ARRL text Image Communications Handbook ${ }^{7}$ describes the legacy of the over five decades of Amateur Radio analog and digital image communications and current projects.

\section{From Faculty Supervisor to Station Trustee}

The involvement of a faculty supervisor is crucial to the eventual establishment of an Amateur Radio resource for the ECE capstone senior design project. The faculty responsible for the curriculum in digital data and wireless communication and electromagnetic wave propagation are the obvious sponsors of the effort to establish an Amateur Radio station as a laboratory facility of the ECE department and to become the station trustee. Faculty without an Amateur Radio license can readily obtain an entry-level license without a Morse Code element. With an Amateur Radio license the university-based station can then be licensed to the faculty supervisor as the trustee.

As an example of the development process, the Temple University Amateur Radio Club (TUARC) K3TU (www.temple.edu/k3tu) has become not only an undergraduate student organization open to the general University populace, but also a laboratory of the undergraduate curriculum in analog and digital communication and electromagnetic waves, which is supported by the Department of Electrical and Computer Engineering (www.temple.edu/ece). The high frequency (HF: 1.8 to $30 \mathrm{MHz}$ ) and the very, ultra and super high frequency (VHF, UHF, SHF: $50 \mathrm{MHz}$ to $2.4 \mathrm{GHz}$ ) communication station equipment (Figure 1) and antennas were provided by the ECE Department as a modest part of the undergraduate communication laboratory.
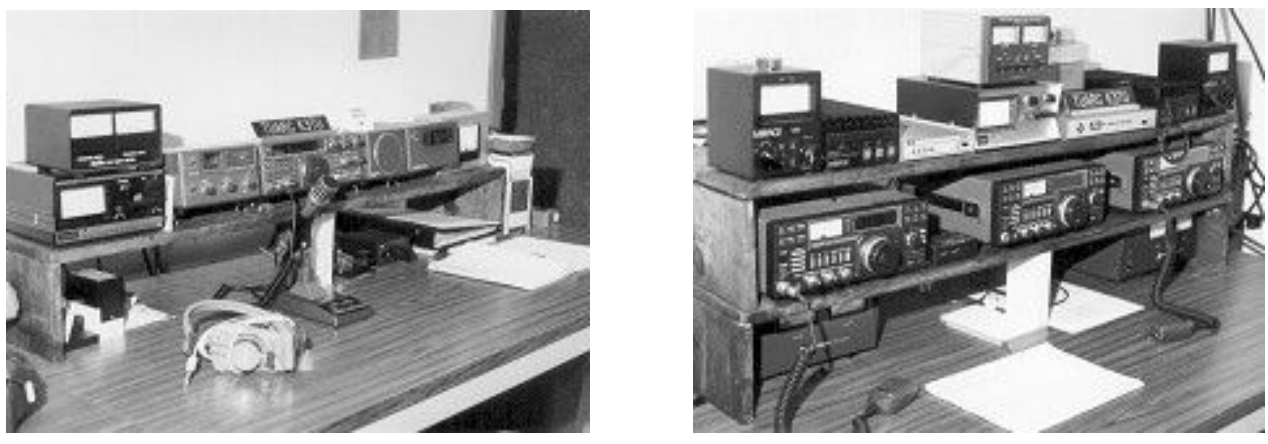

Figure 1. HF (left) and VHF/UHF/SHF (right) communication station equipment at TUARC K3TU. 


\section{ECE Capstone Projects that Resonate with Industry}

The wide expanse of ECE capstone design projects available in digital wireless, microwave and satellite communications and DSP with Amateur Radio serves the undergraduate student well by the resonance that occurs with the industrial sector. These projects obviously also utilize other undergraduate courses other than analog and digital communications, especially electronics, digital logic, DSP and electromagnetic wave propagation.

One of the first ECE capstone design projects that utilized Amateur Radio (1986) was an experimental system for a complex modulation method in that era. A frequency hopping spread spectrum modulation (FHSS) system was developed in the Amateur Radio UHF (420 MHz) band (Figure 2). The ECE undergraduate students were able to successfully demonstrate the digital data transmission of keyboard characters in the presence of a swept interfering carrier frequency.
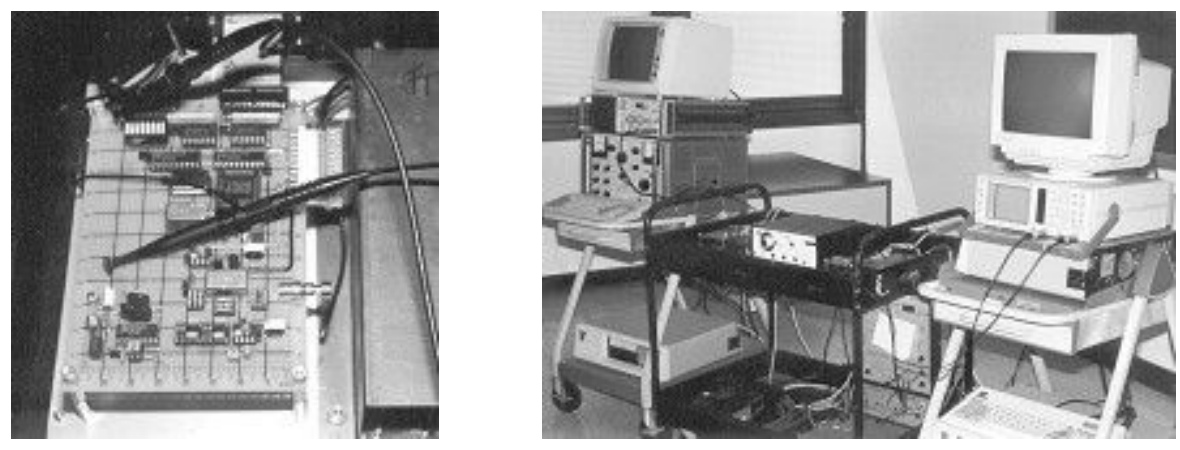

Figure 2. Frequency Hopping Spread Spectrum (FHSS) ECE capstone design project (1986).

Amateur Radio experimentation in the SHF and microwave bands has a long tradition. Early generations of microwave engineers acquired much of their technical training by such activities. Another ECE capstone design project (1990) was a high-speed for the era (2 Mb/sec), $10 \mathrm{GHz}$ carrier frequency, frequency shift keyed (FSK), digital data communication system (Figure 3). Gunn diode transceivers (known as Gunnplexers) were used with automatic frequency control (AFC). Recognizing that this was over a decade ago, the system was demonstrated by a then high-speed data transmission across the University campus, between the Engineering and Computer Science buildings.

Several ECE capstone design projects at Temple University have utilized the Low Earth Orbiting (LEO) Amateur Radio satellites (AMSAT, www.amsat.org). Two such early projects were a microcomputer based azimuth-elevation antenna tracking system (1988) and an automatic Doppler frequency shift correction for phase shift keyed (PSK) data communication (1989) using these LEO satellites. These projects were awarded two successive IEEE Bendix Awards for Region 2, which is given for the outstanding ECE capstone design project.

ECE capstone design projects have continued in this area, with the development of satellite PSK DSP data modems (1996 and 1997) and a complete refurbishing (2002) of the AMSAT crosspolarized, azimuth-elevation VHF/UHF antenna system (Figure 4). This ECE capstone design 

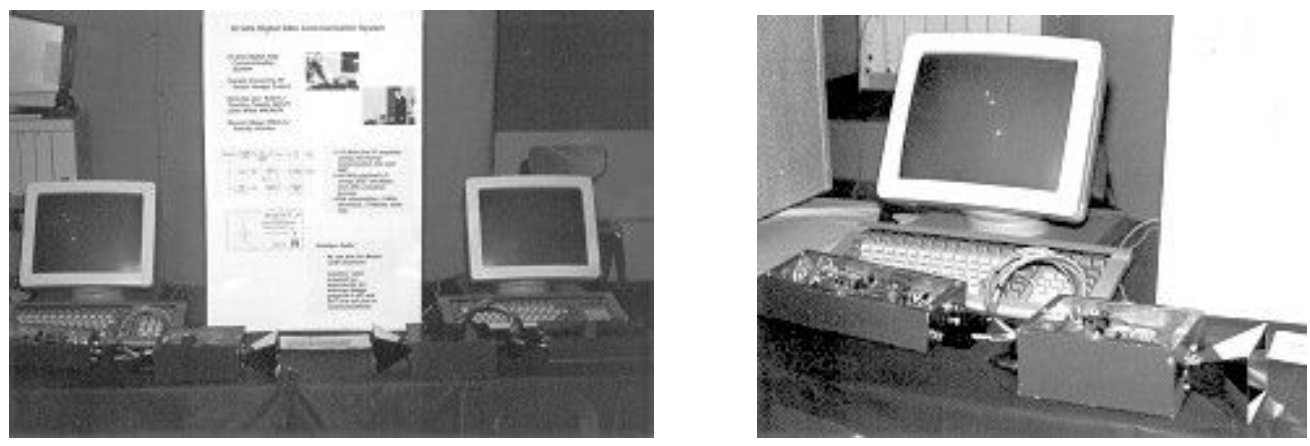

Figure 3. High-speed, $10 \mathrm{GHz}$ digital data communication system ECE capstone design project (1990)

project also recertified the digital data telemetry capabilities of the TUARC K3TU satellite station and was awarded the Best ECE Senior Design Project 2002 by the Department. This interesting project was featured on the local ABC television station (WPVI).
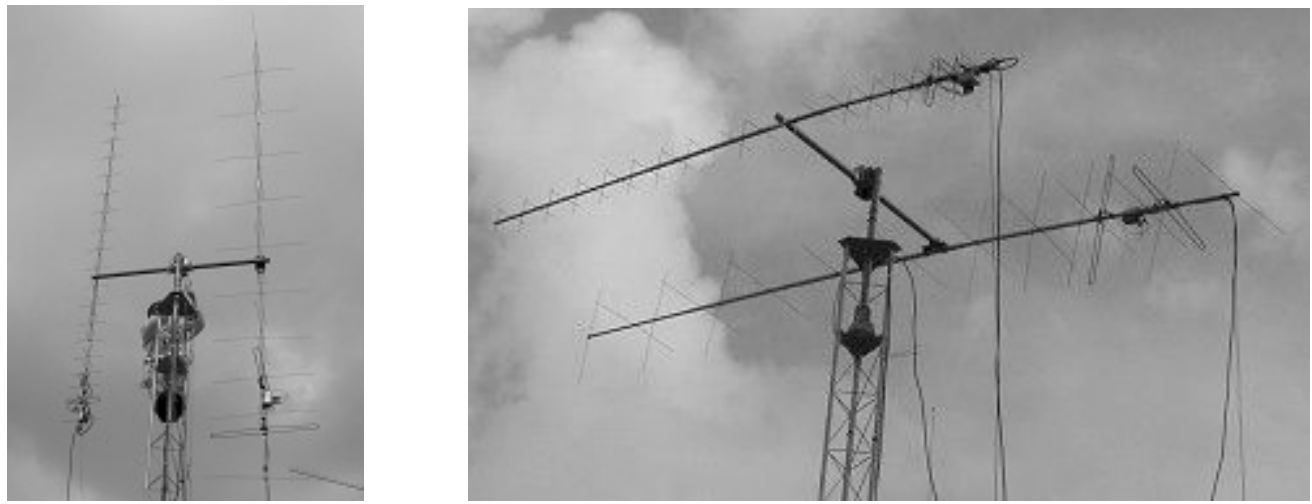

Figure 4. AMSAT antenna refurbishing, part of the ECE capstone design project (2002).

A recent ECE capstone design project utilizing Amateur Radio (2003) has developed data packet protocols for a voice encoder-decoder (vocoder). Although Amateur Radio has fostered a variety of technical advances in communication, notably single-sideband suppressed carrier modulation (SSB-SC), analog amplitude and frequency modulation (AM, FM) continues to be used. This digital voice project (www.temple.edu/k3tu/digital_voice.htm) has been well received by the Amateur Radio community and is now being considered as the emerging standard for digital voice transmission.

The device utilizes a commercial Advanced Multiband Excitation vocoder (the AMBE-2020, $w w w . d v s i . c o m)$ in a DSP microcomputer, but with framed data protocols and processor control that were developed as a part of this ECE capstone design project. The real-time protocols are executed by a host microprocessor (Figure 5, lower left). This ECE capstone design project was published and presented at the TAPR/ARRL Digital Communication Conference ${ }^{8}$, which 
demonstrates that even undergraduate ECE students can add significantly to the base of knowledge in Amateur Radio.

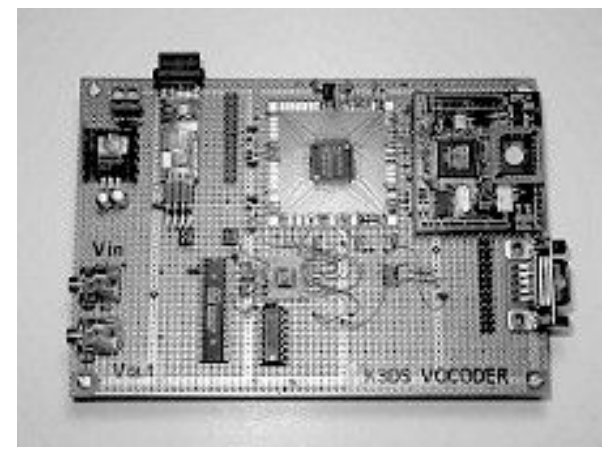

Figure 5. Digital voice vocoder (2003) and OFDM modem (2004) ECE capstone design projects.

The next generation of this ECE capstone design project (2004) is developing an orthogonal frequency division multiplexed (OFDM) modem by interfacing and programming a DSP microcomputer conceived by experimenters in Amateur Radio (Figure 5, upper right).

This low-cost DSP module is the core technology for a variety of projects, including the technical development of a software-defined radio (www.tapr.org). The DSP module is based on the 80 MIPS Analog Devices ADSP-2185N (www.analog.com). An interface board, with 16-bit analog-to-digital and digital-to-analog converters, is available that was also developed by Amateur Radio experimenters (Figure 6). This ADSP-2185N module and interface could also be used in an ECE undergraduate laboratory course in DSP and demonstrates the continuing technical contributions of Amateur Radio.

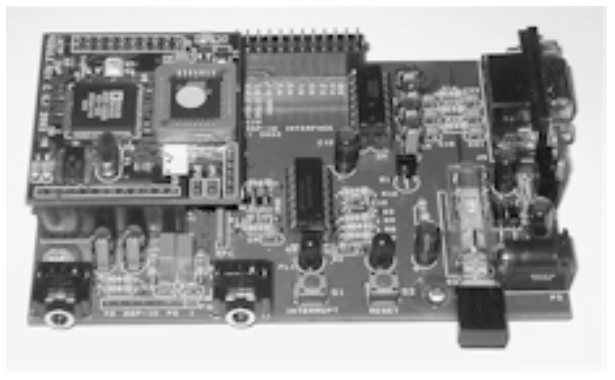

Figure 6. Amateur Radio ADSP-2185N DSP module and interface.

\section{Better Projects, Better Students}

ECE capstone design projects utilizing Amateur Radio are exciting and can be thought of as going beyond the areas that are usually undertaken by undergraduate students. A supportive department and faculty can thus provide an environment for ECE capstone design opportunities in the emerging technologies of the wireless world. Thus, hopefully, the impediments to the reintroduction of Amateur Radio in the ECE capstone design program have been dispelled. The 
technical rewards, for the department, faculty and the undergraduate students, are certainly wide band.

1. QEX Forum for Communications Experimenters. ARRL, Newington, CT (bimonthly).

2. ARRL Handbook for Radio Amateurs. ARRL, Newington, CT (2003).

3. Andre Kesteloot and Charles Hutchinson, Spread Spectrum Sourcebook. ARRL, Newington, CT (2002).

4. Martin Davidoff, Radio Amateur's Satellite Handbook. ARRL, Newington, CT (2002).

5. Douglas Smith, Digital Signal Processing Technology. ARRL, Newington, CT (2001).

6. Thomas McDermott, Wireless Digital Communications: Design and Theory. TAPR, Tucson, AZ (1996).

7. Ralph Taggart, Image Communications Handbook. ARRL, Newington, CT (2002).

8. Melinda Gleiter, Jodi Moore, Yazime Allen, and Dennis Silage, Vocoder Redux: the AMBE 2020. Proc. TAPR/ARRL DCC, 2003.

DENNIS SILAGE (K3DS, silage@ @ temple.edu) received the PhD in EE from the University of Pennsylvania in 1975. He is a Professor, teaches digital data communication and digital signal processing, and is the trustee of the Temple University Amateur Radio Club (K3TU, www.temple.edu/k3tu), which he has integrated into the undergraduate communications curriculum. Dr. Silage is a past chair of the Middle Atlantic Section of the ASEE. 